\title{
ASYMPTOTIC VALUES OF SOME CONTINUOUS MAPPINGS
}

\author{
A. CANTÓN AND J. QU
}

(Communicated by Jeremy Tyson)

\begin{abstract}
It is shown that the set of asymptotic values of a light continuous mapping defined on $\mathbb{R}^{s}$ is an analytic set in the sense of Suslin.
\end{abstract}

A mapping $f: \mathbb{R}^{s} \rightarrow \mathbb{R}^{t}$ is light if for every $a \in f\left(\mathbb{R}^{s}\right)$ the preimage $f^{-1}(a):=$ $\left\{x \in \mathbb{R}^{s}: f(x)=a\right\}$ is totally disconnected. A particular instance is a discrete mapping for which every fiber $f^{-1}(a)$ is a discrete set. For example, non-constant holomorphic functions are discrete. If $f: \mathbb{R}^{s} \rightarrow \mathbb{R}^{t}$ is a light continuous mapping, then $s \leq t$ (see [2] and [8).

A point $a \in \mathbb{R}^{t}$ is an asymptotic value of a continuous mapping $f: \mathbb{R}^{s} \rightarrow \mathbb{R}^{t}$ if $f(x) \rightarrow a$ as $|x| \rightarrow \infty$ on some continuous path $\gamma \subset \mathbb{R}^{s}$. The set of asymptotic values of $f$ will be denoted by $\operatorname{As}(f)$.

A Suslin analytic set in $\mathbb{R}^{t}$ is a continuous image of a Borel set. There are several equivalent definitions (see, for example, [3, Lemma 11.6]) but in this note there will be used the characterization of analytic sets (in a complete separable metric space) in terms of the Suslin $\mathcal{A}$-operation. Concretely, $A \subset \mathbb{R}^{t}$ is analytic if and only if

$$
A=\bigcup_{\mathbb{N}^{\mathbb{N}}} \bigcap_{p \geq 1} S_{n_{1}, \ldots, n_{p}},
$$

where the sets $S_{n_{1}, \ldots, n_{p}} \subset \mathbb{R}^{t}$ are closed and $\mathbb{N}^{\mathbb{N}}$ is the collection of all infinite sequences of (positive) natural numbers (see [1, [3, Lemma 11.7] or [7, p. 207]). Sierpinski calls the set $A$ the nucleus of the defining system $\left\{S_{n_{1}, \ldots, n_{p}}\right\}$.

In 4, S. Mazurkiewicz shows that the set of asymptotic values of a holomorphic (or meromorphic) function, $f$, defined in $\mathbb{C}$, is an analytic set. In fact, as pointed out to the authors by Alexandre Eremenko, Mazurkiewicz does not use the analycity of the function $f$. Indeed, he defines a function $\rho_{f}: \mathbb{C} \times \mathbb{C} \rightarrow \mathbb{R}^{+}$as the infimum of the diameters of the $f$-images of curves connecting two points. When $f$ is light $\rho_{f}$ is a distance. Let $\mathcal{X}_{f}^{*}$ denote the completion of the metric space $\mathcal{X}_{f}=\left(\mathbb{C}, \rho_{f}\right)$. Mazurkiewicz observes that the set of asymptotic values of $f$ is the image (under the continuous function $f$ ) of the Borel set $\mathcal{X}_{f}^{*} \backslash \mathcal{X}_{f}$, and hence, analytic. Consequently, Mazurkiewicz's proof and conclusion can be extended to light mappings defined in $\mathbb{R}^{s}$ (with $s \geq 3$ ). An important class of such mappings are non-constant quasiregular

Received by the editors July 10, 2013 and, in revised form, October 18, 2013.

2010 Mathematics Subject Classification. Primary 54C10.

The first author was partially supported by a grant from Ministerio de Ciencia e Innovación (Spain), MTM 2009-07800. The second author performed her research while on leave from the Chinese Academy of Sciences and thanks Purdue University, and, in particular, Professor Drasin for his advice and hospitality. The second author was supported by NSFC Project 11271215. 
mappings which, although in general not smooth, are discrete and therefore light (see [6] or 9]).

In this note, we present an alternative approach to Mazurkiewicz's result, where we use the characterization of analytic sets given by the Suslin $\mathcal{A}$-operation, and work on the range of $f$ rather than on its domain.

Theorem 1. Let $f: \mathbb{R}^{s} \rightarrow \mathbb{R}^{t}(s \leq t)$ be a light continuous mapping. Then, the set of asymptotic values of $f$ is an analytic set.

It will be shown that $\operatorname{As}(f)$ can be written in terms of the Suslin $\mathcal{A}$-operation. In order to do this, consider a dyadic partition of $\mathbb{R}^{t}$. Concretely, let $\left\{X_{n}\right\}_{n \geq 1}$ be the family of closed unit cubes with vertices on the integer lattice so that $\mathbb{R}^{t}=\bigcup_{n>1} X_{n}$. Each $X_{n}$ is divided into $2^{t}$ congruent closed cubes $X_{n_{1}, n_{2}}$ with $n_{2} \in\left\{1, \ldots, 2^{t}\right\}$ having side length $1 / 2$. In general, a dyadic cube $X_{n_{1}, n_{2}, \ldots, n_{p}}$ of side length $2^{1-p}$ is divided into $2^{t}$ congruent closed cubes of side length $2^{-p}$, and each of these cubes is denoted by $X_{n_{1}, n_{2}, \ldots, n_{p}, n_{p+1}}$ with $n_{p+1} \in\left\{1,2, \ldots, 2^{t}\right\}$. For $p \geq 1$, let $\mathcal{F}_{p}$ be the family of all dyadic cubes of generation $p$, that is, $\mathcal{F}_{p}=\left\{X_{n_{1}, n_{2}, \ldots, n_{p}}: n_{1} \in \mathbb{N}, n_{i} \in\right.$ $\left.\left\{1, \ldots, 2^{t}\right\}, 2 \leq i \leq p\right\}$. For $X_{n_{1}, n_{2}, \ldots, n_{p}} \in \mathcal{F}_{p}(p \geq 1)$, consider the set

$$
\operatorname{Adj}\left(X_{n_{1}, n_{2}, \ldots, n_{p}}\right)=\left\{x \in X: X \in \mathcal{F}_{p}, X \cap X_{n_{1}, n_{2}, \ldots, n_{p}} \neq \varnothing\right\},
$$

consisting of the cube together with its neighbors of generation $p$. Clearly, $X_{n_{1}, n_{2}, \ldots, n_{p}}$ lies in the interior of $\operatorname{Adj}\left(X_{n_{1}, n_{2}, \ldots, n_{p}}\right)$.

Given a light continuous mapping $f: \mathbb{R}^{s} \rightarrow \mathbb{R}^{t}$, a dyadic cube $X_{n_{1}, n_{2}, \ldots, n_{p}}$ is said to be admissible if $f^{-1}\left(\operatorname{Adj}\left(X_{n_{1}, n_{2}, \ldots, n_{p}}\right)\right)$ has an unbounded connected component. For any finite sequence of natural numbers, $n_{1}, \ldots, n_{p}$, define

$$
S_{n_{1}, n_{2}, \ldots, n_{p}}= \begin{cases}X_{n_{1}, \ldots, n_{p}}, & \text { if } X_{n_{1}, \ldots, n_{p}} \text { is admissible } \\ \varnothing, & \text { otherwise }\end{cases}
$$

Notice that $S_{n_{1}, \ldots, n_{p}}$ is closed, with diameter $\operatorname{diam}\left(S_{n_{1}, \ldots, n_{p}}\right) \leq 2^{1-p} \sqrt{t}$ and $S_{n_{1}, \ldots, n_{p}, n_{p+1}} \subset S_{n_{1}, \ldots, n_{p}}$. Theorem 1 is a consequence of Proposition 1

Proposition 1. Let $f: \mathbb{R}^{s} \rightarrow \mathbb{R}^{t}(s \leq t)$ be a light continuous mapping and $A$ the analytic set that is the nucleus of the defining system $\left\{S_{n_{1}, \ldots, n_{p}}\right\}$ given by (1). Then,

$$
A=\operatorname{As}(f) \backslash\{\infty\} .
$$

Remark 1. If $A$ is analytic, then $A \cup\{\infty\}$ is also analytic.

Proof. First it will be shown that $A \subset \operatorname{As}(f) \backslash\{\infty\}$ or, in words, that any point $a \in A$ is a finite asymptotic value of $f$. Since $a \in A$ there exists a sequence of natural numbers $\left\{n_{p}\right\}_{p \geq 1}$ such that $a=\bigcap_{p \geq 1} S_{n_{1}, \ldots, n_{p}}$ with $S_{n_{1}, \ldots, n_{p}} \neq \varnothing$ for all $p \geq 1$. Thus for every $p \geq 1, S_{n_{1}, \ldots, n_{p}} \stackrel{=}{=} X_{n_{1}, \ldots, n_{p}}$ where $n_{2}, \ldots, n_{p} \in$ $\left\{1, \ldots, 2^{t}\right\}$ and $X_{n_{1}, \ldots, n_{p}}$ is admissible and therefore $f^{-1}\left(\operatorname{Adj}\left(X_{n_{1}, \ldots, n_{p}}\right)\right)$ has an unbounded connected component. Given $k \in \mathbb{N}$ there is $p \in \mathbb{N}$ such that $B(a, 1 / k) \supset$ $\operatorname{Adj}\left(X_{n_{1}, \ldots, n_{p}}\right)$, where $B(a, r)$ denotes the open ball of radius $r>0$ centered at $a$. Since $f^{-1}\left(\operatorname{Adj}\left(X_{n_{1}, \ldots, n_{p}}\right)\right)$ has an unbounded connected component $f^{-1}(B(a, 1 / k))$ 
has an unbounded connected component. Therefore there exists a chain

$$
C_{1} \supset C_{2} \supset \cdots \supset C_{k} \supset \cdots
$$

of nested sets where each $C_{k}$ is the closure of an unbounded connected component of $f^{-1}(B(a, 1 / k))$. The continuity of $f$ implies that each $C_{k}(k \geq 1)$ is path connected.

Since $f$ is light there can be found an increasing subsequence of natural numbers, $\left\{k_{j}\right\}_{j \geq 1}$, and a strictly increasing sequence of positive real numbers, $\left\{r_{j}\right\}_{j \geq 0}$, with $r_{j} \nearrow \infty(j \rightarrow \infty)$ such that for any $j \in \mathbb{N}$,

a) $C_{k_{j}} \cap \overline{B\left(r_{j-1}\right)}=\varnothing$, and,

b) $C_{k_{j}} \cap S\left(r_{j}\right) \neq \varnothing$,

(where $B(r)=B(0, r), S(r)=\partial B(r)$ and $\bar{A}$ is the closure of the set $A$ ). Otherwise, there exists $\rho>0$ such that $C_{k} \cap B(\rho) \neq \varnothing$ for all $k \geq 1$. Consider $\Omega=\bigcap_{k \geq 1} C_{k}$ a subset of $f^{-1}(a)$. Since $\left\{C_{k}\right\}_{k \geq 1}$ is a decreasing sequence of unbounded closed sets that intersect $S(\rho), \Omega \neq \varnothing$, and moreover, $\Omega \cap S(r) \neq \varnothing$ for any $r \geq \rho$. Let $\widehat{\mathbb{R}^{s}}=\mathbb{R}^{s} \cup\{\infty\}$ be the Alexandroff one-point compactification of $\mathbb{R}^{s}$. Since every $C_{k}, k \geq 1$, is an unbounded connected closed set, $\widehat{C_{k}}=C_{k} \cup\{\infty\}$ is a connected compact set in $\widehat{\mathbb{R}^{s}}$ and $\left\{\widehat{C_{k}}\right\}_{k \geq 1}$ decreases to $\widehat{\Omega}=\Omega \cup\{\infty\}$ where $\widehat{\Omega}$ is compact and connected with more than one point. Hence by [5, Corollaire XIII], $\widehat{\Omega}$ can be decomposed as $\widehat{\Omega}=U \cup V$ with $U$ and $V$ disjoint connected sets, each containing more than one point. Let $U$ be the set that contains $\infty$; then $V \subset \mathbb{R}^{s}$ is a connected subset of $f^{-1}(a)$ with more than one point, which contradicts the assumption that $f$ is light.

Hence we can use a) and b) above to find an asymptotic path for $a$. Pick $x_{1} \in C_{k_{1}} \cap S\left(r_{1}\right)$ and let $\gamma_{1}$ be a continuous path in $C_{k_{1}}$ that joins $x_{1}$ with $C_{k_{2}} \cap S\left(r_{2}\right)$. Let $x_{2} \in C_{k_{2}} \cap S\left(r_{2}\right)$ be the other end of $\gamma_{1}$. In general for $j \geq 2$, if $x_{j} \in C_{k_{j}} \cap S\left(r_{j}\right)$ let $\gamma_{j}$ be a continuous path in $C_{k_{j}}$ that joins $x_{j}$ with $C_{k_{j+1}} \cap S\left(r_{j+1}\right)$ and define $x_{j+1}$ to be the other end of $\gamma_{j}$. Let $\gamma=\bigcup_{j \geq 1} \gamma_{j}$. By construction, $\gamma$ is a continuous curve and $\gamma \rightarrow \infty$. Indeed, for any $r>0$ there exists $j \in \mathbb{N}$ such that $r_{j}>r$ and by condition a) above

$$
\bigcup_{i \geq j+1} \gamma_{i} \subset C_{k_{j+1}} \subset\left\{|x|>r_{j}\right\} \subset\{|x|>r\}
$$

Finally it remains to show that $\gamma$ is an asymptotic curve with $a$ as an asymptotic value. Let $\varepsilon>0$ and choose $j \in \mathbb{N}$ so that $1 / k_{j}<\varepsilon$. Take $x \in \bigcup_{i \geq j+1} \gamma_{i} \subset \gamma$. Then $x \in C_{k_{j+1}} \subset \overline{f^{-1}\left(B\left(a, 1 / k_{j+1}\right)\right)} \subset f^{-1}\left(\overline{B\left(a, 1 / k_{j}\right)}\right)$ and therefore $f(x) \in$ $\overline{B\left(a, 1 / k_{j}\right)}$, that is, $|a-f(x)| \leq 1 / k_{j}<\varepsilon$, as desired.

Next there will be shown the other inclusion, that is, that any finite asymptotic value of $f$ belongs to the set $A$. Let $b \in \operatorname{As}(f) \backslash\{\infty\}$ and consider the family of dyadic cubes such that $b=\bigcap_{p \geq 1} X_{n_{1}, \ldots, n_{p}}$. Then, for any $p \geq 1$, there is an $\varepsilon>0$ so that $b \in B(b, \varepsilon) \subset \operatorname{Adj}\left(X_{n_{1}, \ldots, n_{p}}\right)$ since $b \in \operatorname{int}\left(\operatorname{Adj}\left(X_{n_{1}, \ldots, n_{p}}\right)\right)$. Since $b$ is a finite asymptotic value of $f, f^{-1}(B(b, \varepsilon))$ has an unbounded connected component and therefore the set $f^{-1}\left(\operatorname{Adj}\left(X_{n_{1}, \ldots, n_{p}}\right)\right)$ also has an unbounded connected component. Thus $X_{n_{1}, \ldots, n_{p}}$ is admissible and therefore $S_{n_{1}, \ldots, n_{p}}=X_{n_{1}, \ldots, n_{p}}$ for every $p \geq 1$ which implies $b=\bigcap_{p \geq 1} S_{n_{1}, \ldots, n_{p}}$; that is, $b \in A$. 


\section{ACKNOWLEDGEMENTS}

The authors thank David Drasin and Alexandre Eremenko for their helpful comments and suggestions.

\section{REFERENCES}

[1] Lennart Carleson, Selected problems on exceptional sets, Van Nostrand Mathematical Studies, No. 13, D. Van Nostrand Co., Inc., Princeton, N.J.-Toronto, Ont.-London, 1967. MR0225986 (37 \#1576)

[2] P. T. Church, Discrete maps on manifolds, Michigan Math. J. 25 (1978), no. 3, 351-357. MR512905 (80g:57014)

[3] Thomas Jech, Set theory, Springer Monographs in Mathematics, Springer-Verlag, Berlin, 2003. The third millennium edition, revised and expanded. MR.1940513 (2004g:03071)

[4] S. Mazurkiewicz, Sur les points singuliers d'une fonction analytique, Fund. Math. 17 (1931), 26-29.

[5] B. Knaster and C. Kuratowski, Sur les ensembles connexes, Fund. Math. 2 (1921), 206-255.

[6] Seppo Rickman, Quasiregular mappings, Ergebnisse der Mathematik und ihrer Grenzgebiete (3) [Results in Mathematics and Related Areas (3)], vol. 26, Springer-Verlag, Berlin, 1993. MR:1238941 (95g:30026)

[7] W. Sierpinski, Introduction to General Topology, University of Toronto Press, Toronto, 1934.

[8] Jussi Väisälä, Local topological properties of countable mappings, Duke Math. J. 41 (1974), 541-546. MR0350688 (50 \#3180)

[9] Matti Vuorinen, Conformal geometry and quasiregular mappings, Lecture Notes in Mathematics, vol. 1319, Springer-Verlag, Berlin, 1988. MR950174(89k:30021)

Departamento de Ciencias Aplicadas a la Ingeniería Naval, Universidad Politécnica De Madrid, Avda. Arco de la Victoria 4, 28040 Madrid, Spain

E-mail address: alicia.canton@upm.es

Academy of Mathematics and System Science, Chinese Academy of Sciences, No. 55 East Zhongguancun Road, Beijing 100190, People's Republic of China

E-mail address: qu11@math.purdue.edu

Current address: Jinchunyuan West Building, Mathematical Science Center, Tsinghua University, Beijing 100080, People's Republic of China

E-mail address: quijingjing@amss.ac.cn 